\section{Kein Sulfasalazin bei Antibiotika-Allergie!}

\author{
Sulfonamid-basierte Arzneimittel umfassen neben Antibiotika auch \\ Nicht-Antibiotika wie Diuretika, Antidiabetika und Analgetika. Zur \\ Risikoabschätzung von IgE-Kreuzreaktionen ist jedoch nicht die Indi- \\ kation des Sulfonamids entscheidend, sondern die chemische Struktur.
}

\begin{abstract}
tudien zeigen, dass Patienten mit einer Allergie gegen SulfonamidAntibiotika in der Regel kein erhöhtes Allergierisiko gegenüber den strukturchemisch abweichenden sulfonamidischen Nicht-Antibiotika haben. Es gibt allerdings Ausnahmen: Dazu zählt Sulfasalazin, das als antiinflammatorischer Wirkstoff z.B. bei rheumatischen Erkrankungen oder entzündlichen Darmerkrankungen eingesetzt wird. Die chemische Struktur dieses nicht-antibiotischen Sulfonamids ist der von Sulfonamid-Antibiotika sehr ähnlich und zahlreiche schwere aller-
\end{abstract}

gische Reaktionen gegen die Substanz sind beschrieben.

In einem In-vitro-Experiment sollte nun die Kreuzreaktivität zwischen einem antibakteriell wirksamen Sulfonamid und Sulfasalazin untersucht werden. Dazu wurden mononukleäre Zellen des peripheren Blutes von zwei Patienten mit einer Sulfasalazin-Sensibilisierung sowie von drei Patienten mit einer Sulfamethoxazol-Allergie zusammen mit den Seren einer gesunden Kontrollgruppe einem Lymphozytentransformationstest unterzogen. Dabei reagierten die mononukleären Zellen von beiden Patienten- gruppen sowohl auf Sulfapyridin, den aktiven Metaboliten von Sulfasalazin, als auch auf Sulfamethoxazol. Dieser Befund lässt auf eine starke Kreuzreaktivität der Sulfonamide schließen. Auf die Bestätigung durch einen Provokationstest bei den durchgängig schwer allergisch reagierenden Patienten wurde aber verzichtet.

Fazit: Sulfonamid-Antibiotika und das antiinflammatorisch wirksame Sulfasalazin rufen starke immunologische Kreuzreaktionen hervor. Deshalb sollte bei Vorliegen einer Allergie gegen eine der Substanzen die jeweils andere kreuzreagierende Substanz gemieden werden.

Barbara Kreutzkamp

Zawodniak A et al. Cross-reactivity in drug hypersensitivity reactions to sulfasalazine and sulfamethoxazole. Int Arch Allergy Immunol 2010; 153: 152-6

\section{Bitterstoffe als neue Asthmatherapie?}

Rezeptoren für bitteren Geschmack dienen der Toxinerkennung in Nahrung und Atemluft. Bei der Untersuchung der von Bitterstoffen ausgelösten Signaltransferkaskade und den physiologischen Reaktionen machten Forscher jetzt eine überraschende Entdeckung.

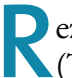
ezeptoren für bitteren Geschmack (TAS2Rs) findet man auf der Zunge sowie auf der glatten Muskulatur der Atemwege. Dies führte ein interdisziplinäres Forscherteam aus Maryland, USA, zunächst zu der Hypothese, dass bei bestimmten bronchospastischen Erkrankungen wie z. B. Asthma und chronisch obstruktiver Lungenerkrankung (COPD), inhalative Substanzen über die Stimulation der TAS2Rs atemwegsverengend wirken könnten.

TAS2R-Agonisten wie Saccharin, Chloroquin und Denatonium - die bitterste bekannte Substanz - führen zu einem intrazellulären Anstieg von Kalziumionen. In Experimenten mit isolierter glatter Muskulatur der Atemwege löste die bitterstoffinduzierte intrazelluläre $\mathrm{Ca}^{2+}$-Ausschüttung aus dem sarkoplasmatischen Retikulum jedoch nicht, wie erwartet, eine Muskelkonstriktion aus. Stattdessen bewirkte sie eine Relaxa- tion und Dilatation der Atemwege, die dreimal so stark ausfiel wie bei Einsatz von betaadrenergen Rezeptoragonisten. Die TAS2R-induzierte Relaxation erfolgt aufgrund einer Membran-Hyper-

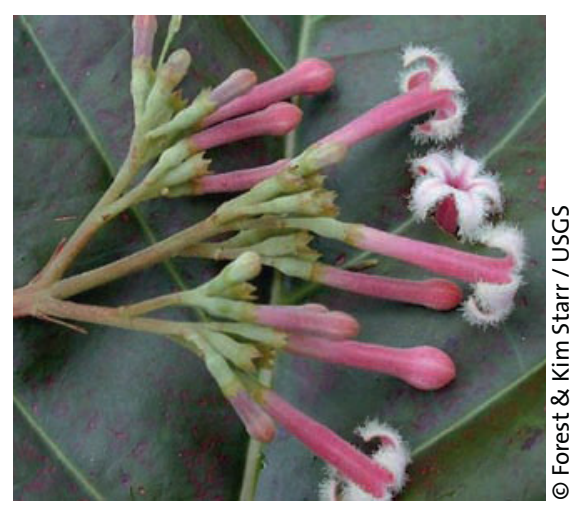

Der südamerikanische Chinarindenbaum gehört zur Ordnung der enzianartigen Gewächse. Sein bitterer Rindeninhaltsstoff Chinin senkt nicht nur Fieber, sondern erweitert auch die Bronchien bei Asthma. polarisation der glatten Muskulatur der Atemwege, vermittelt durch Öffnung kalziumaktivierter Kaliumkanäle mit hoher Leitfähigkeit (,large-conductance $\mathrm{Ca}^{2+}$ activated $\mathrm{K}^{+}\left(\mathrm{BK}_{\mathrm{C}}\right)$ channels"). Entsprechend der unterschiedlichen Wirkmechanismen waren mit Bitterstoffen und Betaagonisten additive Effekte zu erzielen.

Auch im intakten murinen Asthma-Modell ging durch Inhalation von Bitterstoffen die Atemwegskontraktion deutlich zurück. Dabei war eine Dosis von $150 \mu \mathrm{g}$ Chinin $3 \mu \mathrm{g}$ des meistverwendeten Betaagonisten Albuterol bei sensibilisierten Tieren sogar überlegen.

Fazit: Inhalierte Bitterstoffe relaxieren die glatten Muskelzellen der Atemwege und wirken im murinen Asthma-Modell stärker bronchodilatatorisch als Betaagonisten. Auch ein kombinierter Einsatz beider Wirkstoffgruppen ist denkbar.

Barbara Kreutzkamp

Deshpande DA et al. Bitter taste receptors on airway smooth muscle bronchodilate by localized calcium signaling and reverse obstruction. Nat Med 2010; 16 : 1299-1305 